\title{
Glycogen metabolism in Schistosoma mansoni worms after their isolation from the host
}

\author{
Aloysius G.M. Tielens and Simon G. van den Bergh \\ Laboratory of Veterinary Biochemistry, State Universiry of Utrecht, Utrecht, The Netherlands
}

(Received 8 December 1986; accepted 18 March 1987)

\begin{abstract}
Adult Schistosoma mansoni worms rapidly degrade their endogenous glycogen stores immediately after isolation from the host. In NCTC 109 or in a diphasic culture medium the glycogen levels slowly recovered again after the initial decrease. The rapid degradation of glycogen could be prevented, even in a simple salt medium, if $100 \mathrm{mM}$ glucose and $1 \%$ bovine serum albumin were present. Incubations with ${ }^{14} \mathrm{C}$-labelled glucose under different conditions revealed that the degradation of glycogen was induced by the limited catabolism of external glucose. Conditions are described which induce glycogen degradation or resynthesis by $S$. mansoni. The physiological function of the glycogen stores is probably to provide substrate during periods of insufficient supply of external glucose. It is speculated that such periods occur when the worm pair moves into the small mesenteric veins of the host. This hypothesis explains the remarkable wandering behaviour of the parasite in the mesenteric veins, since the schistosomes would have to return to larger vessels when their endogenous glycogen stores are exhausted.
\end{abstract}

Key words: Schistosoma mansoni; Energy metabolism; In vitro culture; Glycogen

\section{Introduction}

Adult Schistosoma mansoni worms contain glycogen which can be rapidly degraded, mainly to lactate $[1,2]$. These - especially in the male - large glycogen stores are often taken for granted, although their function is still not understood and rather intriguing. In intestinal parasites, glycogen stores act as a major energy reserve to span the time between the meals of the host. This function seems superfluous, however, in the adult $S$. mansoni, which lives in the bloodstream of a mammalian host. There, the glucostatic mechanisms of the host guarantee a continuous supply of glucose to the parasite. On the other hand, the pres-

Correspondence address: Dr. A.G.M. Tielens, Lab. Vet. Biochem., P.O. Box 80 177, 3508 TD Utrecht, The Netherlands.

Abbreviations: BSA, bovine serum albumin; $S_{5}, S_{100}$, $\mathrm{S}_{2}^{+}, \mathrm{S}_{5}^{+}, \mathrm{S}_{100}^{+}$, simple salt solutions supplemented with BSA and containing glucose (subscript shows glucose concentration in $\mathrm{mM}$ and superscript + indicates presence of $1 \%$ BSA); HEPES, 4-(2-hydroxyethyl)-1-piperazine ethanesulfonic acid. ence of glycogen reserves which could be shown to be metabolically active suggests that the parasite in certain conditions needs more glucose than it can obtain at that moment from the blood of the host. In this paper, a new hypothesis on the physiological function of the glycogen stores of $S$. mansoni will be discussed.

It has been known for a long time that incubations of $S$. mansoni in vitro inevitably result in the rapid degradation of the endogenous glycogen reserves. This phenomenon occurs in all media described in the literature and with all known isolation procedures even when glucose is provided at normal homeostatic concentrations (see, for instance, refs. 1-6). This rapid degradation of glycogen interferes with all investigations of the carbohydrate metabolism of isolated schistosomes and, therefore, has been further investigated in our laboratory.

Conditions are described here under which $S$. mansoni worms, after an initial breakdown, replenish their glycogen reserves. Furthermore, it was established that a simple salt medium supplemented with bovine serum albumin and contain- 
ing $100 \mathrm{mM}$ glucose, prevents a decrease in glycogen levels during 5-h incubations. It was also shown that the rapid decrease in glycogen content of $S$. mansoni after its isolation is not caused by the uncontrolled degradation of glycogen. The breakdown of endogenous glycogen seems to be connected with the inability of the parasite to utilize sufficient external glucose.

\section{Materials and Methods}

Chemicals. Bovine serum albumin (BSA) was purchased from Boehringer (Mannheim, F.R.G.), delipidated with active carbon and subsequently dialysed before use. All enzymes and fine chemicals were from Boehringer (Mannheim, F.R.G.), the Nutrient Agar was from Difco (Detroit, MI, U.S.A.) and the NCTC 135 (for the preparation of NCTC 109) was from Serva (Heidelberg, F.R.G.). D- $\left[6-{ }^{14} \mathrm{C}\right]$ glucose was obtained from New England Nuclear (Boston, MA, U.S.A.).

Parasites. $S$. mansoni worms were isolated from ether-anaesthetized hamsters $45-50$ days after infection. For all experiments, intact worm pairs were used. Unless stated otherwise, the isolation was done by perfusion via the heart and/or the aorta at $37^{\circ} \mathrm{C}$ with a medium, indicated as $\mathrm{S}_{20}(\mathrm{~S}$ standing for simple salt solution and the subscript showing the glucose concentration). This medium contained $20 \mathrm{mM}$ HEPES, $85 \mathrm{mM} \mathrm{NaCl}, 5.4$ $\mathrm{mM} \mathrm{KCl}, 0.7 \mathrm{mM} \mathrm{Na}_{2} \mathrm{HPO}_{4}, 1 \mathrm{mM} \mathrm{MgSO}, 1.5$ $\mathrm{mM} \mathrm{CaCl}, 25 \mathrm{mM} \mathrm{NaHCO}_{3}, 200 \mathrm{mg} \mathrm{l}^{-1}$ streptomycin, $200 \mathrm{IU} \mathrm{ml}^{-1}$ penicillin and $20 \mathrm{mM}$ glucose $(\mathrm{pH} 7.4)$.

Incubations. In experiments in which the glycogen content of $S$. mansoni was measured (Figs. 1-3; Table 1) 3 worm pairs were incubated in closed $25-\mathrm{ml}$ Erlenmeyer flasks in a gyratory shaker at $37^{\circ} \mathrm{C}$ in $5 \mathrm{ml}$ of the incubation medium. All incubations were started immediately after the isolation of the worms. The gas phase was $95 \%$ $\mathrm{O}_{2}$ plus $5 \% \mathrm{CO}_{2}$. If the glucose concentration in a medium was varied, the $\mathrm{NaCl}$ concentration was adjusted in order to maintain the original osmolarity. For that purpose also NCTC 135 without $\mathrm{NaCl}$ was purchased.

A diphasic culture system was prepared con- sisting of Difco nutrient agar (70\%) with fresh, defibrinated and inactivated rabbit blood (30\%), which is overlaid with Hanks' balanced salt solution [7].

The incubations were terminated by removing the parasites from the incubation medium. The worm pairs were quickly rinsed in $0.9 \% \mathrm{NaCl}$ and $\mathrm{HClO}_{4}$ was added to a final concentration of $5 \%$. Thereafter, the worms were rinsed again with $0.9 \% \mathrm{NaCl}$ and dissolved in $0.5 \mathrm{M} \mathrm{KOH}, 100 \mu \mathrm{l}$ per worm pair.

Radioactive incubations. Twenty-five worms pairs were incubated for $3 \mathrm{~h}$ in $5 \mathrm{ml}$ medium in the presence of $\mathrm{D}-\left[6-{ }^{14} \mathrm{C}\right]$ glucose: $30 \mu \mathrm{Ci}$ in incubations containing $100 \mathrm{mM}$ glucose and $3 \mu \mathrm{Ci}$ in those with $5 \mathrm{mM}$ glucose. A 1 -h preincubation of the worms in $\mathrm{S}_{100}^{+}$(the superscript ${ }^{+}$indicating the presence of $1 \% \mathrm{BSA}$ ) preceded all the radioactive incubations. The incubations were terminated by adding, through the rubber stopper, 200 $\mu l 6 \mathrm{M} \mathrm{KOH}$ to a removable centre well and 60 $\mu l 12 \mathrm{M} \mathrm{HCl}$ to the medium. After $2 \mathrm{~h}$ the worms were isolated and dissolved in $0.5 \mathrm{M} \mathrm{KOH}, 100$ $\mu l$ per worm pair. To precipitate the BSA in the supernatants, if present, $\mathrm{HClO}_{4}$ was added to the medium (final concentration 5\%). The supernatant was then neutralized and used for the biochemical analyses.

Assays. All the carbon dioxide was trapped in the centre well by shaking for another $2 \mathrm{~h}$ after the termination of the radioactive incubations. The radioactivity in this fraction was counted in Tritosol, modified according to Pande [8]. This scintillation medium was also used for all other radioactive compounds in these experiments.

Lactate was separated from the neutralized incubation medium on an acid-washed alumina $\left(\mathrm{Al}_{2} \mathrm{O}_{3}\right)$ column $(2 \mathrm{~g}, h=3.5 \mathrm{~cm})$ which was eluted with $\mathrm{H}_{2} \mathrm{O}(50 \mathrm{ml})$ to isolate glucose, whereupon the alumina was dispersed in $1.5 \mathrm{ml}$ of a quaternary ammonium hydroxide ('Lumasolve', Lumac Systems AG, Basel, Switzerland). After the addition of scintillation medium the radioactivity in this mixture was counted. The total of labelled and unlabelled lactate was determined in the neutralized incubation medium with a standard enzymatic method [9]. 
Glycogen was determined by comparison of the amount of glucose present in neutralized aliquots of a homogenate of the worms in $0.5 \mathrm{M} \mathrm{KOH}$ before and after enzymatic hydrolysis in $0.25 \mathrm{M}$ phosphate buffer ( $\mathrm{pH} \mathrm{6)}$ with $2.2 \mathrm{U} \mathrm{ml}^{-1}$ amyloglucosidase (EC 3.2.1.3). The glycogen content of the worms after the incubations was compared with the glycogen content of worms taken immediately after the isolation, but otherwise treated in exactly the same way. It was verified that this rapid and sensitive enzymatic determination of the glycogen levels in schistosomes gave the same results as ethanol precipitations of glycogen.

Glucose was assayed enzymatically according to a standard procedure [10].

Protein was determined with the Lowry method, using BSA as standard. All glycogen and protein assays were performed in duplicate.

\section{Results}

Recovery of glycogen content after an initial breakdown. Preliminary experiments had shown that during in vitro incubations of $S$. mansoni, even in very rich media, a rapid decline in glycogen levels occurred immediately after the isolation of the parasite. We observed this drop, for example, in a diphasic culture system (further indicated as 'diphasic medium') and in NCTC 109, two media in which egg-laying schistosomes can

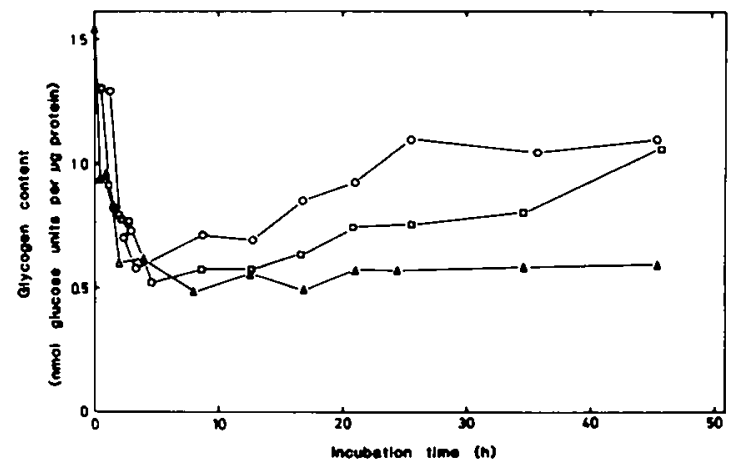

Fig. 1. Glycogen levels of $S$. mansoni worm pairs during in vitro incubations in 3 different media. The parasites were isolated in $S_{5}$ from 2 hamsters, which were infected identically. The worms were pooled and incubations containing 4 worm pairs were started in a diphasic culture medium $(0)$, NCTC $109(\square)$, or $S_{S}(\triangle)$. Each point is the mean of two separate incubations. be maintained for many days $[7,11]$. The inadequacy of these media for the maintenance of the glycogen stores was further investigated in prolonged (48-h) incubations.

Fig. 1 is an example of a representative experiment and shows that after isolation the endogenous glycogen stores were rapidly degraded in a simple salt solution that contained $5 \mathrm{mM}$ glucose $\left(S_{5}\right)$ as well as in the very rich media, NCTC 109 and diphasic medium. However, after this initial decrease, the glycogen levels of the worms incubated in NCTC 109 or diphasic medium increased again. In $S_{5}$, on the other hand, there was no restoration of the glycogen levels.

These experiments were performed with worms which were isolated with $S_{5}$ via a heart perfusion of ether-anaesthetized hamsters. Changes in the isolation medium or procedure had no effect: using NCTC 109 instead of $S_{5}$ as isolation buffer (not shown) and also the use of different methods for the isolation always resulted in the same pattern of glycogen degradation and resynthesis (Fig. 2).

The average protein content $(54 \mu \mathrm{g})$ of the worm pairs after $48 \mathrm{~h}$ incubation in diphasic medium (Fig. 2) was $98 \%$ of that at the beginning.

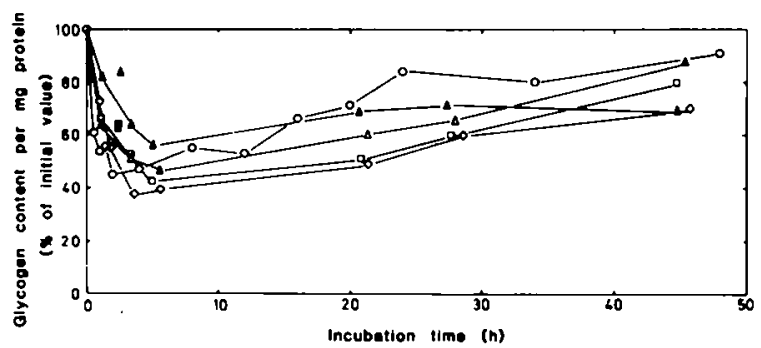

Fig. 2. The effect of various isolation techniques on the glycogen levels of $S$. mansoni worm pairs during incubations in a diphasic culture medium. The parasites were isolated: (a) by perfusion with $S_{5}$ of hamsters treated with $\mathrm{CO}_{2}(\diamond)$, Fentanyl citrate, Fluanisone and diazepam ( $\square$ ), ether (O); or killed by cervical dislocation $(\Delta)$, or (b) by allowing the worms to crawl out of infected veins during the incubation $(\Delta)$. These veins were obtained from a hamster which received 400 I.E. heparin (i.p.) $0.5 \mathrm{~h}$ prior to cervical dislocation. Each point is the mean of two separate incubations, containing 3 worm pairs each. The glycogen levels per $\mathrm{mg}$ protein are expressed as a percentage of the corresponding value of worms collected immediately after the isolation. 


\section{TABLE I}

Effect of various concentrations of glucose and BSA in the incubation medium on the maintenance of glycogen levels by $S$. mansoni worm pairs

\begin{tabular}{lll}
\hline $\begin{array}{l}\text { Glucose con- } \\
\text { centration } \\
(\mathrm{mM})\end{array}$ & $\begin{array}{l}\text { BSA concentration } \\
\left(\mathrm{g}(100 \mathrm{ml})^{-1}\right)\end{array}$ & $\begin{array}{l}\text { Glycogen levels after } \\
5 \mathrm{~h}(\% \text { of initial value })\end{array}$ \\
\hline 5 & 0 & $53 \pm 5(3)$ \\
5 & 1 & $52 \pm 4(2)$ \\
20 & 0 & $58 \pm 8(3)$ \\
20 & 1 & $80 \pm 7(4)$ \\
100 & 0 & $74 \pm 8(5)$ \\
100 & 0.04 & $86 \pm 5(2)$ \\
100 & 0.2 & $88 \pm 4(2)$ \\
100 & 1 & $93 \pm 8(7)$ \\
\hline
\end{tabular}

The parasites were isolated by perfusion with $S_{20}$, and immediately incubated in the simple salt solution for $5 \mathrm{~h}$. Each value is the mean \pm SD of completely independent experiments, the number of which is given in brackets. In all experiments 2-5 separate incubations with 3 worm pairs were carried out. The glycogen content per $\mathrm{mg}$ protein is expressed as a percentage of the corrresponding value of worms collected immediately after the isolation.

Prevention of glycogenolysis by BSA and very high glucose concentrations. Further experiments were done to try and prevent the degradation of glycogen that occurred even in the very rich media, NCTC 109 and diphasic medium. It was discovered that a simple salt solution was sufficient for the maintenance of the glycogen stores in $S$. mansoni, if only the medium contained a very high glucose concentration and was supplemented with BSA. NCTC 109 and diphasic medium contained $5.6 \mathrm{mM}$ glucose. The effects of various concentrations of glucose and BSA are shown in Table I. These data show that a glucose concentration of $100 \mathrm{mM}$ plus the presence of $1 \%$ BSA almost completely prevented glycogenolysis during 5-h incubations. In the presence of $100 \mathrm{mM}$ glucose and $1 \%$ BSA a further enrichment of the incubation medium (by replacing the simple salt solution with NCTC 109) had no positive effect on the maintenance of the glycogen levels (data not shown). On the other hand, a continuous-flow system, for the refreshment of the incubation medium, resulted in slightly but significantly higher levels of glycogen. A continuous flow $\left(20 \mathrm{ml} \mathrm{h}^{-1}\right)$ of $\mathrm{S}_{100}^{+}$resulted after $5 \mathrm{~h}$ incubation in an average of $98 \%$ of the glycogen content at zero-time ( 3 independent experiments, not shown).
The presence of BSA in the incubation medium not only had a positive effect on the maintenance of the glycogen levels, but also on the general condition of the worms. In contrast to incubations without BSA, the parasites always remained paired in the presence of BSA, and they firmly attached themselves to the bottom of the incubation Erlenmeyer flasks with their suckers. This positive effect of BSA on the general condition of the parasites was independent of its effect on the glycogen metabolism: worms in incubations with $S_{5}^{+}$, which resulted in an extensive loss of glycogen, remained paired and in perfect condition.

The protein content of the worms incubated in $\mathrm{S}_{100}^{+}$remained constant during the 5-h incubations. The average protein content of the worm pairs of the 7 independent experiments in $S_{100}^{+}$ presented in Table I was $98 \%$ of the average protein content of control worm pairs immediately after their isolation from the same hamsters.

The influence of proteins other than BSA on the rapid glycogenolysis after isolation was tested, but in the presence of $1 \%$ ovalbumin, $1 \%$ hemoglobin or a collagen sponge, a poorer maintenance of the glycogen levels was observed than in the presence of $1 \%$ BSA (data not shown). Human serum albumin was as effective as BSA.

Manipulation of glycogen metabolism. By varying the glucose concentration of the incubation medium, glycogen metabolism could be manipulated (Fig. 3). A glucose concentration of $100 \mathrm{mM}$ resulted in the maintenance of the original glycogen levels, whereas low glucose concentrations always resulted in a net degradation of glycogen. Furthermore, a condition during which a net synthesis of glycogen occurred, could be induced by further incubating the parasites in buffer with a high glucose concentration $\left(S_{100}^{+}\right)$after the depletion of the glycogen stores in $\mathrm{S}_{2}^{+}$a buffer with a low glucose concentration (Fig. 3 ).

Interaction between the glucose and glycogen metabolism. To investigate this interaction, incubations of $S$. mansoni were performed both in $\mathrm{S}_{5}^{+}$, which resulted in a significant decrease of the endogenous glycogen stores, as well as in $S_{100}^{+}$when practically only externally present glucose was 
TABLE II

Glucose and glycogen metabolism of $S$. mansoni worm pairs

\begin{tabular}{|c|c|c|c|c|c|c|}
\hline \multirow[t]{2}{*}{ Hamster } & \multirow[t]{2}{*}{ Incubation medium } & \multirow[t]{2}{*}{ Glycogen content" } & \multirow[t]{2}{*}{ Glycogen degraded ${ }^{b}$} & \multicolumn{3}{|c|}{$\begin{array}{l}\text { End products } \\
\left.\text { (nmol h }^{-1}(\mathrm{mg} \text { protein })^{-1}\right)\end{array}$} \\
\hline & & & & ${ }^{14} \mathrm{CO}_{2}$ & ${ }^{14} \mathrm{C}$-lactate & total lactate \\
\hline $\begin{array}{l}1 \\
2\end{array}$ & $\begin{array}{l}S_{100}^{+} \\
S_{100}^{+}\end{array}$ & $\begin{array}{l}1.45 \\
1.49\end{array}$ & $\begin{array}{l}13 \\
27\end{array}$ & $\begin{array}{l}138 \\
125\end{array}$ & $\begin{array}{l}1628 \\
1576\end{array}$ & $\begin{array}{l}1723 \\
1696\end{array}$ \\
\hline $\begin{array}{l}1 \\
2\end{array}$ & $\begin{array}{l}\mathrm{S}_{\mathrm{s}}^{+} \\
\mathrm{S}_{5}^{+}\end{array}$ & $\begin{array}{l}1.05 \\
1.16\end{array}$ & $\begin{array}{l}280 \\
247\end{array}$ & $\begin{array}{r}82 \\
116 \\
\end{array}$ & $\begin{array}{r}974 \\
1201 \\
\end{array}$ & $\begin{array}{l}1312 \\
1569\end{array}$ \\
\hline
\end{tabular}

The parasites were incubated for $3 \mathrm{~h}$ in the presence of $\mathrm{D}-\left[6-{ }^{14} \mathrm{C}\right]$ glucose. The end products were analysed in the worm-free medium. All values are corrected for blank incubations.

"At the end of the radioactive incubation in $\mu \mathrm{mol}$ glucose units (mg protein) $)^{-1}$.

${ }^{b}$ In $\mathrm{C}_{3}$ units $\mathrm{h}^{-1}$ (mg protein $)^{-1}$.

metabolized. The presence of BSA had no effect on the glucose metabolism of $S$. mansoni: the endproduct formation in $\mathrm{S}_{5}^{+}$(Table II) was similar to that in $S_{5}[12]$.

Table II shows that the degradation of glycogen, which occurred in $\mathrm{S}_{5}^{+}$was accompanied by a lower rate of degradation of external glucose as compared to the degradation of glucose in $S_{100}^{+}$ when no significant glycogenolysis occurred. Apparently, the degradation of glycogen does not result in an increased glycolytic flux.

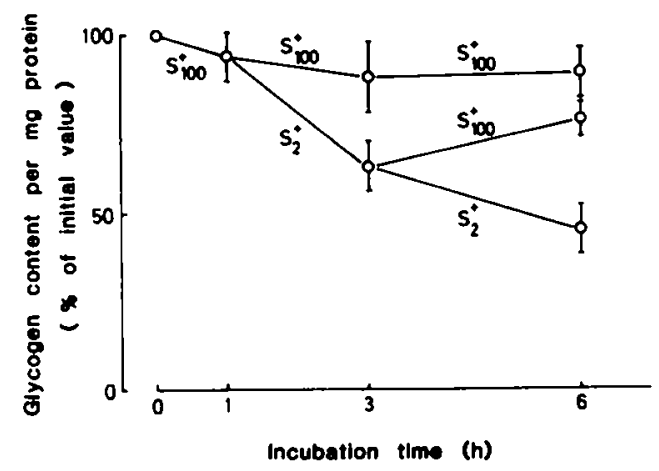

Fig. 3. Manipulation of the glycogen metabolism of S. mansoni by the subsequent use of different glucose concentrations in the incubation medium. The parasites were isolated by perfusion with $S_{20}$ and subsequently incubated in the media indicated in the figure. Each point is the mean \pm SD of 9 separate incubations containing 4 worm pairs each. The glycogen content of the worm pairs is expressed as a percentage of the corresponding value of worms collected immediately after the isolation.

\section{Discussion}

The large glycogen reserves of $S$. mansoni were rapidly degraded after the isolation of the parasite in media with low glucose content (Fig. 1). This occurred even in diphasic medium, in which egg-producing worm pairs can be cultured for many days [7]. After 1-2 days the glycogen levels of worms in this medium and of those in NCTC 109 were restored, whereas in a simple salt solution the glycogen levels remained low (Fig. 1). NCTC and diphasic medium are suitable to achieve a recovery of the glycogen levels and, therefore, could be expected to be also rich enough to prevent a drop in glycogen in the first place. However, substituting NCTC 109 for $S_{20}$ as isolation medium to prevent even the shortest contact of the worms with an inadequate buffer, did not prevent the rapid degradation of glycogen (not shown). Apparently, it was not the inadequacy of the medium, but something else in the procedure that caused the immediate degradation of glycogen. To investigate the effect of the isolation procedure itself, several procedures were tested. However, 4 different treatments of the hamster before the perfusion via the heart, all resulted in the same pattern of glucose degradation and resynthesis (Fig. 2). Even omission of the perfusion by allowing the worm pairs to crawl out of little pieces of vein during the incubations, gave no significant improvement of the maintenance of the glycogen levels (Fig. 2).

Meaningful studies on the biochemistry of $S$. mansoni cannot be performed when the carbohydrate metabolism of the worms is disturbed. 
This disturbance is indicated by the rapid breakdown of the endogenous glycogen stores. Therefore, more vigorous methods were tried to keep the glycogen degradation within acceptable limits. It turned out that a very high glucose concentration $(100 \mathrm{mM})$ plus the presence of $1 \% \mathrm{BSA}$ in a simple salt solution almost completely prevented the glycogenolysis that was up to then always observed after the isolation of the parasites (Table I). The effect of BSA was twofold: it improved not only the maintenance of the glycogen levels, but also the general condition of the worms. The positive effect on the maintenance of glycogen levels is probably caused by the adsorptive power of the protein. If the protein was to be used as substrate, then the presence of amino acids, as in NCTC, should have been beneficial too, and this was not the case. Furthermore, a continuous refreshment of the incubation medium resulted in even higher levels of glycogen than those presented in Table I. This indicates, to our mind, that BSA did not act as substrate but, most likely, as a scavenger of 'toxic' substances.

The results obtained in this study with NCTC 109 and the diphasic culture system compare favorably with those reported in the literature in other rich media. For instance in Medium 199 supplemented with $10 \%$ newborn calf serum no recovery of the glycogen levels of $S$. mansoni occurred [4]. It is remarkable that in this medium no influence of high glucose concentrations on the maintenance of the glycogen stores could be demonstrated [4].

The discovery of the effects of the glucose concentration and the presence of BSA led to the possibility of manipulating the glycogen metabolism of schistosomes (Fig. 3). Comparison of incubations when glycogenolysis occurred with those when the glycogen levels remained constant, revealed that the degradation of glycogen did not result in an increased glycolytic flux. The glucose and glycogen degradation acted in concert: glycogenolysis occurred when the degradation of external glucose was reduced (Table II). Therefore, the degradation of glycogen is not just an uncontrolled action, caused by the isolation procedure, but the result of a restricted catabolism of external glucose.
The physiological function of the endogenous glycogen of $S$. mansoni is still unknown, but at least a large part of the glycogen can be metabolized [1-6]. The presence of metabolically active glycogen strongly indicates that the parasite sometimes needs an energy reserve: i.e. moments exist when the externally available glucose is not sufficient for a normal functioning. At first we considered the possibility that at the normal glucostatic value of the blood-glucose concentration in the host, the rate of glucose uptake is insufficient to keep up with the normal requirement of the schistosome. The worm would (partly) consume its endogenous glycogen stores when the blood-glucose concentration of the host is at its normal venous value. But it would replenish its stores by synthesizing glycogen after the meals of the host, when the glucose concentrations in the portal and mesenteric veins of the host (the natural habitat of adult $S$. mansoni) increases several-fold over its normal homeostatic value. However, our experiments showed that this is unlikely to be correct, because even in $5.6 \mathrm{mM}$ glucose the glycogen reserves could be replenished (Fig. 1). Furthermore, exceptions are known of $S$. mansoni living in other parts of the bloodstream, for instance in the brain, where severalfold increases of the homeostatic glucose concentration never occur [13].

Another possibility could be that the parasite needs a glycogen reserve for bursts of activity, for example during wandering in the veins. The stored glycogen could be used for the muscle activity during these movements. This would explain the much larger quantity of glycogen in the male as compared to the female, since the male acts as a vehicle for the female. The movement of a worm pair into a small mesenteric vein could even have a more profound effect on the degradation of glycogen than only the expenditure of the costs of transportation. A comparison of the dimensions of a small mesenteric vein with those of a $S$. mansoni worm pair, in our opinion, reveals that the flow of blood through a vein occupied by a worm pair will be severely restricted. This will limit the amount of glucose available to these worms and they will have to consume their endogenous glycogen stores. By the time their glycogen reserves are exhausted the parasites will 
have to move downstream towards the liver into larger veins (including the portal vein) to replenish their glycogen stores. This hypothesis on periods with insufficient external substrate would not only explain the presence of the large endogenous glycogen reserves in $S$. mansoni, but it would also provide a possible biochemical explanation for the very frequent movements of worm pairs in vivo. Schistosomes visit many sites per day, and this remarkable wandering behaviour in the veins of the host was demonstrated by the pattern of egg deposition in single-pair infections $[14,15]$. The observed deposition of eggs at far-apart places in the intestinal veins is exactly what one would expect if the worms alternately move downstream towards the liver and upstream again into the smaller veins. If the blood flow through an infected vein is indeed severely restricted, this would also imply that the parasite intermittently lives in a more or less anaerobic environment. The forced, intermittent anaerobic functioning would explain why schistosomes rely on a predominantly anaerobic energy metabolism. This anaerobic metabolism has always been an enigma, because up to now schistosomes were supposed to live in an aerobic habitat.

Our results show that during prolonged incubations in the presence of the normal homeostatic glucose concentration $(5.6 \mathrm{mM})$ the glycogen stores of $S$. mansoni could eventually be replenished (Fig. 1). This supports the hypothesis that the glycogen storage of schistosomes is not meant to bridge the gap in between the meals of the host, but is intermittently used either for extra activity or because the parasite somtimes resides in areas with a lower glucose concentration.

However, the rapid decrease of the glycogen content of $S$. mansoni, which is observed immediately after the isolation from the host, is still unexplained. In our opinion, isolation of the parasites causes damage to the uptake system of glucose. This forces the parasite to live on the endogenous glycogen reserves. In suitable buffers the schistosomes slowly recover from the isolation trauma. It is noteworthy that after the isolation of $S$. mansoni egg-laying also shows a lag phase of a few days [7,11]. This supports our view that the increased capacity for the uptake of glucose is due to a recovery of the worm. As long as the normal uptake of glucose is not restored, a very high glucose concentration is necessary to prevent glycogenolysis (Table I). The necessity of an excessively high glucose concentration indicates that then the main way of glucose uptake is not via a transporter but by passive diffusion.

The knowledge of the described disturbances in the metabolism of glucose and glycogen could shed new light on previous studies on glucose uptake and the transport of glucose from the male to the female, as most of these earlier studies were performed with freshly isolated parasites [16].

The described incubation media, which permit at will the manipulation of glycogen metabolism, make thorough studies of the carbohydrate metabolism of schistosomes feasible, and the improvements of the media might even help to find the missing link in the long-term cultivation of schistosomes.

\section{Acknowledgements}

The authors express their appreciation to $\mathrm{Mr}$. J.M. van den Heuvel, Mr. R.H. Elfring, Mr. M. van de Wetering and Mr. A. Verwijs for their skillful technical assistance, and to Dr. A.M. Deelder and Dr. J.P. Rotmans from the Department of Parasitology, State University of Leiden, for the supply of infected hamsters.

This investigation received financial assistance from the UNDP/World Bank/WHO Special Programme for Research and Training in Tropical Diseases. It was also supported by the Netherlands Foundation for Chemical Research (SON) with financial aid from the Netherlands Organization for the Advancement of Pure Research (ZWO).

\section{References}

1 Bueding, E. (1950) Carbohydrate metabolism of Schistosoma mansoni. J. Gen. Physiol. 33, 475-495.

2 Bueding, E. and Fisher, J. (1982) Metabolic requirements of schistosomes. J. Parasitol. 68, 208-212.

3 Czok, R., Czifer, S. and Jelinic, B. (1975) Glykogenmetabolismus in Schistosoma mansoni Wien. Tierărztl. Monatsschr. 62, 249-254.

4 Mercer, J.G. and Chappell, L.H. (1985) Schistosoma mansoni: effect of maintenance in vitro on the physiology and biochemistry of adult worms. Parasitology 90, 339-349. 
5 Mercer, J.G. and Chappell, L.H. (1986) The effect of maintenance in vitro on glucose uptake and the incorporation of glucose into glycogen by adult Schistosoma mansoni. Int. J. Parasitol. 16, 253-261.

6 Tielens, A.G.M., Houweling, M. and van den Bergh, S.G. (1985) The effect of 5-thioglucose on the energy metabolism of Schistosoma mansoni in vitro. Biochem. Pharmacol. 34, 3369-3373.

7 Schiller, E.L., Bueding, E., Turner, V.M. and Fisher, J. (1975) Aerobic and anaerobic carbohydrate metabolism and egg production of Schistosoma mansoni in vitro. J. Parasitol. 61, 385-389.

8 Pande, S.V. (1976) Liquid scintillation counting of aqueous samples using Triton-containing scintillants. Anal. Biochem. 74, 25-34.

9 Hohorst, H.J. (1970) L-(+)Lactat. Bestimmung mit Lactat-Dehydrogenase und NAD. In: Methoden der Enzymatischen Analyse (Bergmeyer, H.U., ed.), pp. 1425-1429, Verlag Chemie, Weinheim/Bergstr.

10 Bergmeyer, H.U., Bernt, E., Schmidt, F. and Stork, H. (1970) D-Glucose. Bestimmung mit Hexokinase und Glucose-6-phosphat-Dehydrogenase. In: Methoden der Enzymatischen Analyse (Bergmeyer, H.U., ed.), pp. 1163-1165, Verlag Chemie, Weinheim/Bergstr.
11 Senft, A.W. and Senft, D.G. (1962) A chemically defined medium for maintenance of Schistosoma mansoni. J. Parasitol. 48, 551-554.

12 Van Oordt, B.E.P., van den Heuvel, J.M., Tielens, A.G.M. and van den Bergh, S.G. (1985) The energy production of the adult Schistosoma mansoni is for a large part aerobic. Mol. Biochem. Parasitol. 16, 117-126.

13 Bambirra, E.A., de Souza Andrade, J., Cesarini, I., Rodrigues, P.A. and Drummond, C.A.S.A.G (1984) The tumoral form of schistosomiasis: report of a case with cerebellar involvement. Am. J. Trop. Med. Hyg. 33, 76-79.

14 Pellegrino, J. and Coelho, P.M.Z. (1978) Schistosoma mansoni: wandering capacity of a worm couple. J. Parasitol. 64, 181-182.

15 Cheever, A.W. and Duvall, R.H. (1982) Schistosoma japonicum: migration of adult worm pairs within the mesenteric veins of mice. Trans. R. Soc. Trop. Med. Hyg. 76 , 641-645.

16 Cornford, E.M. and Fitzpatrick, A.M. (1985) The mechanism and rate of glucose transfer from male to female schistosomes. Mol. Biochem. Parasitol. 17, 131-141. 